\title{
TOTEM, detector and physics
}

\author{
Francesco Cafagna*, on behalf of the TOTEM Collaboration ${ }^{\dagger}$ \\ INFN Sezione di Bari, Italy \\ E-mail: francesco.cafagna@ba.infn.it
}

\begin{abstract}
The TOTEM (TOTal cross section, Elastic scattering and diffraction dissociation Measurement at the LHC) experiment, located at the interaction point 5 of the LHC, has measured the total, elastic and inelastic proton-proton cross-sections, using a luminosity independent method, based on the optical theorem, in a center-of-mass energy range from 2.76 to $13 \mathrm{TeV}$. The elastic scattering was investigated in a wide range of the squared four-momentum transfer $|t|$ allowing study of the Coulomb-nuclear interference region down to $|t| \sim 8 \times 10^{-4} \mathrm{GeV}^{2}$. This made possible the first measurement of the $\rho$ parameter at $\sqrt{s}=13 \mathrm{TeV}, \rho$ being the ratio between the real and the imaginary part of the nuclear elastic scattering amplitude at $t=0$. This measurement, combined with the total cross-section results, led to the exclusion of all the models classified and published by the COMPETE Collaboration. The results obtained by TOTEM are indeed compatible with predictions of a colourless 3 -gluon bound state exchange in the t-channel of proton-proton elastic scattering, as postulated by alternative theoretical models both in the Regge-like framework and in the modern QCD framework.

In this contribution the TOTEM experiment detectors and results will be described, along with the actual experiment status, the future physics program for the LHC Run 3.
\end{abstract}

The Eighth Annual Conference on Large Hadron Collider Physics-LHCP2020

25-30 May, 2020

online

\footnotetext{
* Speaker.

${ }^{\dagger}$ for collaboration list see http://cern.ch/totem
} 


\section{Introduction}

TOTEM measured the total $p p$ cross-section at the energy of $\sqrt{s}=2.76,7,8$ and $13 \mathrm{TeV}$ $[1,2,3,4]$, using a luminosity independent method and validating it by comparing several methods to determine the total cross-sections $[5,6,7,8,9,10]$. The total cross-section can be obtained via the optical theorem that combines the simultaneous measurements of the total inelastic rate, $N_{\text {inel }}$, and the total nuclear elastic rate, $N_{e l}$, with its extrapolation to the optical point $t=0$. This is achieved with the experimental set-up of TOTEM, which consists of two inelastic telescopes, called $\mathrm{T} 1$ and $\mathrm{T} 2$, to detect charged particles produced in inelastic $p p$ collisions, and Roman Pot stations to detect elastically scattered protons at very small angles [11]. The measured ratio of the elastic and inelastic rates $N_{e l} / N_{\text {inel }}$ allows for the determination of the elastic and inelastic cross-sections as well.

To access the smaller $|t|$-value region, the colliding beams must have a beam divergence of not more than a few micro-radians [12]. This can be obtained by either increasing the beta function value, $\beta^{*}$, or by reducing the beam emittance, $\varepsilon$ (beam divergence $=\sqrt{\varepsilon / \beta^{*}}$ ). With special runs having dedicated beam optics configurations, $\beta^{*}=90,1000,2500 \mathrm{~m}$, TOTEM extended the measurement to $|t|$-values as low as $8 \times 10^{-4} \mathrm{GeV}^{2}$, measuring the differential elastic cross-section over a wide range of $t[5,8,10,13]$. This made the extrapolation of the differential cross-section to the optical point at $t=0$ possible, allowing, for the first time at the LHC, the determination of the elastic scattering cross-section, the total cross-section via the optical theorem as well as the measurement of the $\rho$ parameter [10].

To increase robustness and understanding of the systematic uncertainties, the TOTEM Collaboration, cross-checked the results of its measurements by comparing several independent approaches to determine the total cross-section, proving the reliability of the luminosity independent method. Measurements at $7 \mathrm{TeV}$, were double checked using different methods under the same beam conditions, while the ones at 8 and $13 \mathrm{TeV}$ where checked leveraging different beam and optics conditions.

During the special runs, TOTEM provides minimum and zero bias triggers to the CMS experiment [14], making also possible physics studies in common between the two experiments. The collaboration with CMS gave birth to a joint project: CMS-TOTEM Precision Proton Spectrometer (CT-PPS) [15], designed to take data during the LHC Run 2 standard run with high luminosity. The project collected slightly less than $100 \mathrm{fb}^{-1}$, in the last two years of Run 2 data taken, proving the feasibility of the project [16] that has been integrated into CMS as PPS subsystem, eventually.

\subsection{The TOTEM experiment}

The TOTEM inelastic telescopes, T1 and T2, consist of two arms located symmetrically on both sides of the LHC interaction point 5 (IP5): the T1 telescope with cathode strip chambers (CSC), placed at a distance of $9 \mathrm{~m}$ from IP5, covers the pseudorapidity range $3.1 \leq|\eta| \leq 4.7$. The T2 telescope with gas electron multiplier (GEM) chambers, located at $\pm 13.5 \mathrm{~m}$ from IP5, covers the pseudorapidity range $5.3 \leq|\eta| \leq 6.5$. The pseudorapidity coverage of the two telescopes allowed the detection of $95 \%$ of the inelastic events, including events with diffractive mass down to $3.6 \mathrm{GeV}$. 
For the LHC Run 3, an upgrade of the T2 detector has been scheduled. The new T2 will fit in the reduced space left by the new LHC beamline configuration; it will be based on scintillator slabs read out by silicon photomultipliers (SiPM) [17].

Roman Pot (RP) stations are located symmetrically on both sides of the IP5. During the LHC Run 1, the Roman Pot stations were located at $147 \mathrm{~m}$ (RP147) and $220 \mathrm{~m}$ (RP220) along the LHC beam line. Each station is composed of two units (near and far, in the TOTEM jargon) separated by a distance of about $5 \mathrm{~m}$. A unit consists of 3 RPs, two approaching the outgoing beam vertically, from the top and the bottom, and one horizontally. During LHC Run 1, each RP was equipped with a stack of 10 silicon strip detectors, designed with the specific objective of reducing the insensitive area at the edge facing the beam to only a few tens of micrometers. This edgelessness design permits to measure the proton distance from the beam center, in both coordinates perpendicular to the beam, with a resolution of about $11 \mu \mathrm{m}$ [18]. The movement and the alignment of all pots are monitored with a precision better than $20 \mu \mathrm{m}$ based on track reconstruction and external alignment tools. The long lever arm between the near and the far units allows the determination of the track angle in both projections with a precision of about $5 \mu \mathrm{rad}$. A more detailed description of the TOTEM detector and performance, in this configuration, can be found in [11, 19].

The RP layout for the LHC Run 2, has been modified the RP stations previously installed at $\pm 147 \mathrm{~m}$, from the interaction point, have been relocated at $\pm 210 \mathrm{~m}$, with one of the two units, the far one, tilted around the beam axis by $8^{\circ}$ degree, to improve multi-track resolution[20]. Moreover, two newly designed horizontal RP, have been installed between the two units at $\pm 220 \mathrm{~m}$. All RP have been refurbished and upgraded to stand the increase in luminosity foreseen. This new layout has been proposed to improve the apparatus performances, adding timing detectors, to resolve the pileup of multiple events in the same bunch crossing, and operate the experiment at higher luminosities. This has been fulfilled by TOTEM, for the special runs with low luminosity, installing new timing detectors in a vertical RP $[21,22,23]$. The RP upgrade was a feat that boosted the CT-PPS project. In fact the CT-PPS collaboration installed, in two horizontal RP, new tracking detectors, based on the silicon pixels developed for the CMS tracker upgrade [15], while the diamond timing detectors, developed for the TOTEM upgrade, were installed in the new horizontal ones [22]. At the end, CT-PPS was leveraging four different detector technologies: silicon strips and pixels, and diamond and Ultra Fast Silicon Detector (UFSD) [23], for tracking and timing respectively.

For the LHC Run 3, a consolidation and refurbishment of the mechanics and electronics of the station is in progress. Moreover a new diamond based timing detector package will be installed inside an horizontal RP.

\section{Results}

The cross-sections measured by TOTEM are shown in Figure 1, along with the ones obtained by other experiments at LHC and using cosmic ray measurements (see [4] and references therein).

Given the various methods of measuring elastic and inelastic rates, it was possible to perform completely different measurement of the inelastic, elastic and total cross-sections, comparing the results for a better understanding of the systematic uncertainties and double checks the results.

Thanks to the data collected at this energy with $\beta^{*}=1000 \mathrm{~m}$, it was possible to explore the elastic scattering in the Coulomb Nuclear Interference (CNI) region. For the first time at LHC, 

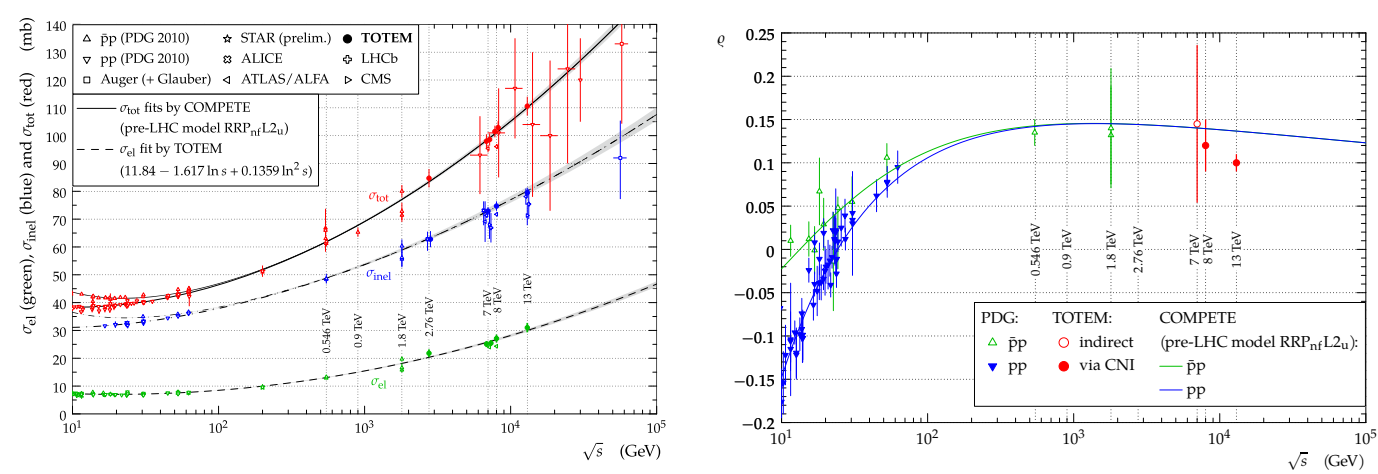

Figure 1: On the left, a compilation (see [4] and references therewith) of $p p$ and $\bar{p} p$ total $\left(\sigma_{t o t}\right)$, inelastic $\left(\sigma_{\text {inel }}\right)$ and elastic $\left(\sigma_{e l}\right)$ cross-sections measurements as a function of $\sqrt{s}$. On th right, the dependence of the $\rho$ parameter on $\sqrt{s}[10,24]$. The $p p$ (blue) and $p \bar{p}$ (green) data are taken from PDG [25].

the $\rho$ parameter was measured, via the CNI, and was found to be $\rho=0.12 \pm 0.03$ [24]. The $\rho$ parameter has been measured also at $\sqrt{s}=13 \mathrm{TeV}$, thanks to the data collected in a special run with $\beta^{*}=2500 \mathrm{~m}$. The values obtained are: $\rho=0.09 \pm 0.01$ and $\rho=0.10 \pm 0.01$, depending on different physics assumptions and mathematical modelling [10]. This time the CNI region has been used to estimate the $\sigma_{t o t}$ using three different and independent approaches. This novel "Coulomb normalization" technique was applied for the first time to LHC data, finding a result of $\sigma_{t o t}=110.3 \pm 3.5 \mathrm{mb}$, that, combined with the luminosity independent method calculation, yields $\sigma_{\text {tot }}=110.5 \pm 2.4 \mathrm{mb}$.

The unprecedented precision of the $\rho$ measurement, shown in Figure 1, combined with the TOTEM total cross-section measurements in an energy range from 2.76 to $13 \mathrm{TeV}$, has implied the exclusion of all the models classified and published by the COMPETE Collaboration [26]. The $\rho$ results obtained by TOTEM are compatible with the predictions, from alternative theoretical models both in the Regge-like framework and in the QCD framework, of a colourless 3-gluon bound state exchange in the $t$-channel of the proton-proton elastic scattering.

\section{Conclusions}

TOTEM has measured, for the first time at the LHC, the total, inelastic and elastic protonproton cross sections at $\sqrt{s}=2.76,7,8$ and $13 \mathrm{TeV}$ using a luminosity independent method. Furthermore, for the first time at LHC, the $\rho$ parameter has been estimated studying the CNI region.

This measurement, combined with the total cross-section results, led to the exclusion of all the models published by the COMPETE Collaboration, and shows compatibility with predictions of a colourless 3-gluon bound state exchange in the t-channel of proton-proton elastic scattering.

Acknowledgment:We are grateful to the beam optics development team for the design and the successful commissioning of the high $\beta^{*}$ optics and to the LHC machine coordinators for scheduling the dedicated fills.

This work was supported by the institutions listed on the front page and also by the Magnus Ehrnrooth foundation (Finland), the Waldemar von Frenckell foundation (Finland), the Academy of Finland, the Finnish Academy of Science and Letters (The Vilho, Yrjö and Kalle Väisälä Fund), the OTKA NK 101438 and the EFOP-3.6.1-16-2016-00001 grants (Hungary). Individuals have received support from Nylands nation vid Helsingfors universitet (Finland), from the MŠMT ČR (Czech Republic) and the János Bolyai Research Scholarship of the Hungarian Academy of Sciences and the NKP-17-4 New National Excellence Program of the Hungarian Ministry of Human Capacities. 


\section{References}

[1] G. Antchev et al. (TOTEM Collaboration), Eur. Phys. J. C, 80 (2020) 91, doi:10.1140/epjc/s10052-020-7654-y.

[2] G. Antchev et al. (TOTEM Collaboration), EPL, 101 (2013) 21004, doi:10.1209/0295-5075/101/21004.

[3] G. Antchev et al., Phys. Rev. Lett. 111 (2013) 012001, doi: 10.1103/PhysRevLett.111.012001.

[4] G. Antchev et al. (TOTEM Collaboration), Eur. Phys. J. C, 79 (2019) 103, doi:10.1140/epjc/s10052-019-6567-0.

[5] G. Antchev et al. (TOTEM Collaboration), EPL, 101 (2013) 21002, doi:10.1209/0295-5075/101/21002.

[6] G. Antchev et al. (TOTEM Collaboration), EPL, 101 (2013) 21003, doi:10.1209/0295-5075/101/21003.

[7] G. Antchev et al. (TOTEM Collaboration), EPL, 96 (2011) 21002, doi: 10.1209/0295-5075/96/21002.

[8] G. Antchev et al. (TOTEM Collaboration), EPL, 95 (2011) 41001, doi: 10.1209/0295-5075/95/41001.

[9] G. Antchev et al. (TOTEM Collaboration), Eur. Phys. J. C, 79 (2019) 861, doi:10.1140/epjc/s10052-019-7346-7.

[10] G. Antchev et al. (TOTEM Collaboration), Eur. Phys. J. C, 80 (2020) 91, doi:10.1140/epjc/s10052-020-7654-y.

[11] G. Anelli et al. (TOTEM Collaboration), JINST, 3, S08007 (2008), doi:10.1088/1748-0221/3/08/S08007.

[12] G. Antchev et al. (TOTEM Collaboration), New J. Phys. 16 (2014) 103041, doi:10.1088/1367-2630/16/10/103041.

[13] G. Antchev et al. (TOTEM Collaboration), Nucl. Phys. B 899 (2015) 527, doi:10.1016/j.nuclphysb.2015.08.010.

[14] CMS Collaboration, JINST 3, S08004 (2008). doi:10.1088/1748-0221/3/08/S08004.

[15] M. Albrow et al. (CMS-TOTEM Collaboration), CMS-TOTEM Precision Proton Spectrometer, CERN-LHCC-2014-021, (2014), url: https://cds.cern.ch/record/1753795 .

[16] A. M. Sirunyan, A. Tumasyan et al., J. High Energ. Phys. , 2018, 153 (2018), doi:10.1007/JHEP07(2018)153.

[17] TOTEM Collaboration, Upgrade of the TOTEM T2 Telescope, Technical Design Report, CERN-LHCC-2019-007, (2019), url: https://cds.cern.ch/record/2677468 .

[18] E. Alagoz et al., JINST, 8, P06009 (2013), doi:10.1088/1748-0221/8/06/P06009.

[19] G. Antchev et al., Int. J. Mod. Phys. A 28, 1330046 (2013), doi: 10.1142/S0217751X13300469.

[20] TOTEM Collaboration, TOTEM Upgrade Proposal, CERN-LHCC-2013-009, (2013), url: https://cds.cern.ch/record/1554299.

[21] TOTEM Collaboration, Timing Measurements in the Vertical Roman Pots of the TOTEM Experiment, CERN-LHCC-2014-020, (2014), url: https://cds.cern.ch/record/1753189 .

[22] G. Antchev et al., JINST, 12, P03007 (2017), doi:10.1088/1748-0221/12/03/P03007. 
[23] M. Berretti et al., JINST, 12, P03024 (2017), doi:10.1088/1748-0221/12/03/P03024.

[24] G. Antchev et al. (TOTEM Collaboration), Eur. Phys. J. C 76 (2016) 661, doi:10.1140/epjc/s10052-016-4399-8.

[25] C. Patrignani et al., (Particle Data Group), Chin. Phys., C40, 100001 (2016).

[26] J. R. Cudell et al. (COMPETE Collaboration), Phys. Rev. Lett. 89, 201801 (2002). 